\title{
Serum osteocalcin: A potential biomarker for primary osteoporosis
}

\author{
Mayur Rai ${ }^{1}$, Tirthal Rai ${ }^{2, *}$, Srinidhi Rai ${ }^{3}$, Janice $\mathrm{D}^{\prime} \mathbf{S a}^{4}$, Parinita $\mathrm{K}^{5}$ \\ ${ }^{\mathbf{1}}$ Associate Professor, Dept. of Orthopaedics, A. J. Institute of Medical Sciences, Mangaluru, Karnataka, ${ }^{\mathbf{2 , 5}}$ Associate Professor, \\ ${ }^{3}$ Assistant Professor, Dept. of Biochemistry, K.S. Hegde Medical Academy, (NITTE University) Mangaluru, Karnataka, \\ ${ }^{4}$ Assistant Professor, Dept. of Biochemistry, Kanachur Medical College, Karnataka, India
}

*Corresponding Author:

Email: tirthalrai@nitte.edu.in

Received: $22^{\text {nd }}$ March, 2018

Accepted: $4^{\text {th }}$ May, 2018

\begin{abstract}
Osteoporosis is a silent killer usually asymptomatic and noticed only after a fracture. Women irrespective of their origin and social strata show an accelerated phase of bone loss, for about 10 yrs after the cessation of ovarian function due to hormonal imbalance. Until now for the diagnosis of osteoporosis Bone mineral density (BMD) and DEXA was used, which is costly hence bone turns over markers have come to vogue in suggesting the risk of osteoporotic fractures.

Objectives of the Study: To assess the potentiality of serum osteocalcin as a diagnostic tool for osteoporosis in post menopausal women and thus overcome the risk of fractures.

Materials and Methods: This study was conducted on 50 apparently healthy pre menopausal women and 50 postmenopausal women with immediate fractures sustained as a result of trivial trauma.

Results: The mean serum osteocalcin level was $(2.63 \pm 1.76)$ in the post menopausal women with fractures as compared to the pre menopausal women $(5.55 \pm 2.92)$ which was statistically significant.

( $\mathrm{p}$ value $<0.001$ ). In the osteoporotic group $40 \%$ were having hip fracture, $34 \%$ were having wrist fracture, $24 \%$ spine and $2 \%$ were having tibia fracture. There was no statistical significance observed in the serum osteocalcin levels among the different fracture groups (p 0.913)

Conclusion: Osteocalcin is a highly sensitive bone formation marker and can be used as a diagnostic or screening tool in post menopausal women for Osteoporosis. The decreased serum osteocalcin levels after menopause could hence predict and prevent the risk of fractures.
\end{abstract}

Keywords: Osteocalcin, Pre-menopausal women, Postmenopausal women with fractures, Calcium, Phosphorous, Alkaline phosphatase, Total protein, Albumin and osteoporosis.

\section{Introduction}

Osteoporosis is a silent killer in elderly age group, usually asymptomatic and noticed only after a fracture, it is characterized by low bone mass and deterioration of bone tissue, leading to weak bones. Osteoporosis is defined as bone mineral density that is less than 2.5SD below the mean peak value in young adults of the same race and sex ( $\mathrm{t}$ score of -2.5$).{ }^{1}$ There are two types of osteoporosis, primary osteoporosis with Type I osteoporosis (postmenopausal), primarily affects the trabecular bone. Vertebral and distal radius fractures are common in these women and type II osteoporosis (SENILE) seen in patients older than 75 years. This affects both cortical and trabecular bone. Hip and pelvic fractures are common in these women. Secondary osteoporosis is due to certain genetic, androgenic, rheumatological, gastrointestinal disorders and drugs like anticonvulsants, glucocorticoids and thyroxine. ${ }^{2}$

Bone grows in size during childhood, with an increase growth during adolescence. The peak bone mass is obtained in adulthood at the age of 35 years, after which it declines with progressive age. Women irrespective of their origin and social strata show an accelerated phase of bone loss, for about $10 \mathrm{yrs}$ after the cessation of ovarian function due to hormonal imbalance. The total risk for women to have an osteoporotic fracture may exceed 30 to $40 \%$ worldwide. $^{2}$

Women have 15 to $18 \%$ lifetime risk of hip fracture after the age of 50 yrs, versus $6 \%$ risk in men. 1 out of 8 males and 1 out of 3 females in India suffer from osteoporosis, making India one of the largest affected countries in the world. Globally osteoporosis is responsible for more than one million fractures per year. $^{3}$

Until now for the diagnosis of osteoporosis, it has been recommended to measure Bone mineral density (BMD) and DEXA, which is unaffordable by the lower strata people. In the last decade or so the bone turns over markers have come to vogue in suggesting the risk of osteoporotic fractures and its treatment modulations. The bone formation marker osteocalcin is synthesized predominantly by the osteoblasts and incorporated into the extracellular matrix (ECM) of bone, osteocalcin is dependent on post - translational carboxylation for its hydroxyapatite affinity, a process that has been shown to decrease with age. High levels of under carboxylated osteocalcin are seen in hip fracture patients and associated with an increased fracture risk, particularly in those over 80 years of age. ${ }^{4}$

Serum osteocalcin is a valid marker of bone turnover when resorption and formation are coupled 
and is a specific marker of bone formation whenever formation and resorption are uncoupled.

The aim of this study was to assess the potentiality of serum osteocalcin as a diagnostic tool for osteoporosis in post menopausal women, in order to overcome the risk of fractures and therefore decrease the morbidity and mortality rate in elderly women.

\section{Materials and Methods}

This study was designed as a case control study which included:

Group I- 50 apparently healthy pre menopausal women Group II -50 postmenopausal women with immediate fractures sustained as a result of trivial trauma. Fractures involving the wrist, spine and hip, (the sample was collected on the day of the fracture.)

The subjects who were excluded were the ones with chronic illness, renal dysfunction, liver disorder, diabetics, immobilised patients, treatment with anticonvulsants and glucocorticoids, surgically induced menopause, Patients on hormone replacement therapy and fractures as a result of road traffic accident/high energy trauma.

An informed consent was obtained from the selected subjects and $5 \mathrm{ml}$ of sample was collected in a plain vacutainer for the biochemical analysis and centrifuged for 10 minutes at 4000rpm and the serum was then analysed for osteocalcin, alkaline phosphatase, calcium, phosphorous, total protein and albumin.

\section{Methods of Estimation}

1. Estimation of serum calcium by Arsenazo method ${ }^{5}$

2. Estimation of serum inorganic phosphorous by phosphomolybdate method ${ }^{6}$

3. Estimation of serum alkaline phosphatase by IFCC / kinetic method $^{7}$

4. Estimation of serum Total Protein by Biuret method $^{8}$

5. Estimation of serum Albumin by Bromocresol Green methodology ${ }^{9}$

6. Estimation of serum osteocalcin by chemiluminescent method ${ }^{10}$

\section{Statistical Analysis}

Student $t$ test was used to compare the quantitative data between the two groups and correlation between variables was done using pearsons test.

One way anova test was used to compare levels among the groups.

Statistical Software: SPSS vs16 was used for the analysis of the data and Microsoft word and Excel have been used to generate graphs, tables etc.

\section{Results}

The study group consisted of 50 apparently healthy pre menopausal women and 50 post menopausal women with immediate fractures due to trivial fall, among the fracture group $40 \%$ were having hip fracture, $34 \%$ were having wrist fracture, $24 \%$ spine and $2 \%$ were having tibia fracture.

The mean serum osteocalcin level was $(2.63 \pm$ $1.76)$ in the post menopausal women with fractures as compared to the pre menopausal women $(5.55 \pm 2.92)$ which was statistically significant. (p value $<0.001$ ) (Table 1)(Fig. 1).

The premenopausal women had statistically significant ( $p$ value $<0.001$ ) higher mean serum calcium level $(9.26 \pm 0.59)$ as compared to the post menopausal women with fractures $(8.63 \pm 0.70)$. (Table 1) (Fig. 2).

The phosphate level had no statistical difference between the two groups. The mean serum phosphorous level in premenopausal women was $(2.74 \pm 0.61)$ and in post menopausal women with fractures it was $(3.02 \pm$ 0.92). (Table 1).

Alkaline phosphatase level was statistically significant as its mean serum level was (105.14 \pm 49.14) in postmenopausal women with fractures as compared to $(74.24 \pm 11.33)$ in pre menopausal women. (Table 1) (Fig. 3).

The mean serum total protein level was not statistically significant in both the study groups whereas the mean serum albumin level was statistically higher $(4.02 \pm 0.18)$ in the premenopausal women as compared to post menopausal group with fractures $(3.68 \pm 0.40)$. (Table 1).

Considering women of both the groups there was a negative correlation of osteocalcin with age ( $\mathrm{r}$ value 0.512 ) ( $\mathrm{p}$ value $<0.001$ ) and was statistically highly significant. (Table 2).

Osteocalcin had a positive correlation with BMI ( $\mathrm{r}$ $0.241, \mathrm{p}-0.016)$. Calcium too had positive correlation and was statistically significant (r 0.261and p- 0.009) whereas there was a negative correlation with phosphorous and was not statistically significant. (Table 2).

There was a negative correlation between osteocalcin and alkaline phosphatase which was highly significant( $\mathrm{r}$ value -0.274 , $\mathrm{p}-0.006$ ). (Table 2)

A negative correlation was seen between osteocalcin and total protein $(r=-0.026, p-0.799)$ which was not significant and a positive correlation between albumin and osteocalcin which was highly significant( $\mathrm{r}$ value 0.260 , $\mathrm{p}$ value 0.009 ). (Table 2 ).

There was no statistical difference observed in serum osteocalcin levels among the different fracture groups (p 0.913) (Table 3). 
Table 1: Comparison of parameters between the pre and postmenopausal women with fracture groups

\begin{tabular}{|l|c|c|c|}
\hline \multicolumn{1}{|c|}{ Parameter } & Postmenopausal with fracture & Premenopausal women & p value \\
\hline Osteocalcin $(\mathrm{ng} / \mathrm{ml})$ & $2.63 \pm 1.76$ & $5.55 \pm 2.92$ & $<0.001$ \\
\hline Calcium $(\mathrm{mg} / \mathrm{dl})$ & $8.63 \pm 0.70(\mathrm{mg} / \mathrm{dl})$ & $9.26 \pm 0.59$ & $<0.001$ \\
\hline Phosphate $(\mathrm{mg} / \mathrm{dl})$ & $3.02 \pm 0.92$ & $2.74 \pm 0.61$ & 0.075 \\
\hline Alkaline Phosphatase(IU/L) & $105.14 \pm 49.14$ & $74.24 \pm 11.33$ & $<0.001$ \\
\hline Total Protein( $\mathrm{g} / \mathrm{dl})$ & $6.32 \pm 0.51$ & $6.49 \pm 0.42$ & 0.076 \\
\hline Albumin $(\mathrm{g} / \mathrm{dl})$ & $3.68 \pm 0.40$ & $4.02 \pm 0.18$ & $<0.001$ \\
\hline
\end{tabular}

Table 2: Correlation of osteocalcin with all the other parameters

\begin{tabular}{|l|c|c|}
\hline Considering both the groups $(\mathbf{n = 1 0 0})$ Osteocalcin vs & R value & P value \\
\hline Age & -0.512 & $<0.001$ \\
\hline BMI & 0.241 & 0.016 \\
\hline Calcium & 0.261 & 0.009 \\
\hline Phosphate & -0.185 & 0.066 \\
\hline Alkaline Phosphatase & -0.274 & 0.006 \\
\hline Total Protein & -0.026 & 0.799 \\
\hline Albumin & 0.260 & 0.009 \\
\hline
\end{tabular}

Table 3: Serum osteocalcin levels in various types of fractures among postmenopausal women

\begin{tabular}{|l|c|c|c|c|c|c|}
\hline Fracture & $\mathbf{N}$ & Minimum & Maximum & Mean & Std. Deviation & p value \\
\hline Hip & 20 & 2.00 & 11.00 & 2.67 & 1.99 & 0.913 \\
\hline Wrist & 17 & 2.00 & 11.00 & 2.72 & 2.15 & \\
\hline Spine & 12 & 2.00 & 2.96 & 2.44 & 0.37 & \\
\hline
\end{tabular}

TIBIA fracture group is excluded* less number

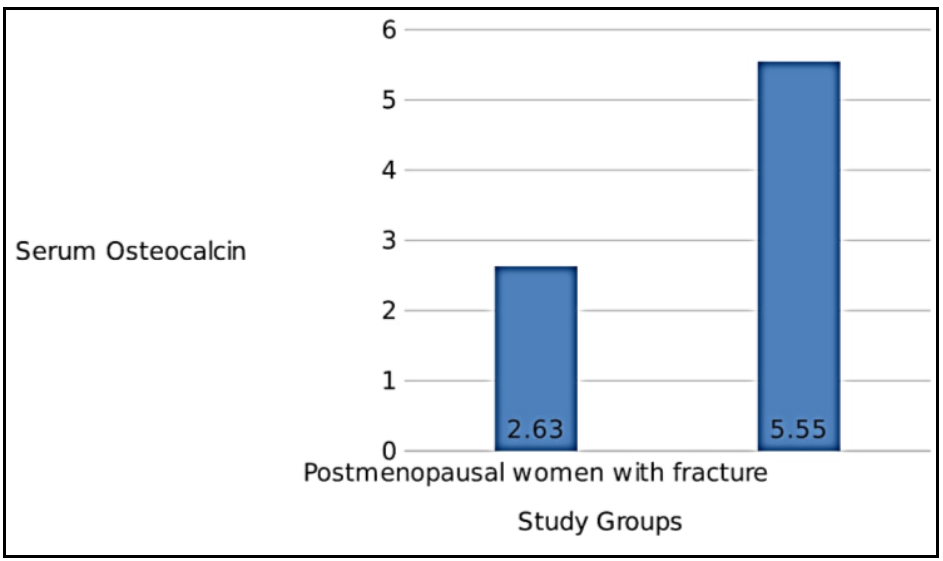

Fig. 1: The mean serum osteocalcin levels in the premenopausal and postmenopausal women with fracture

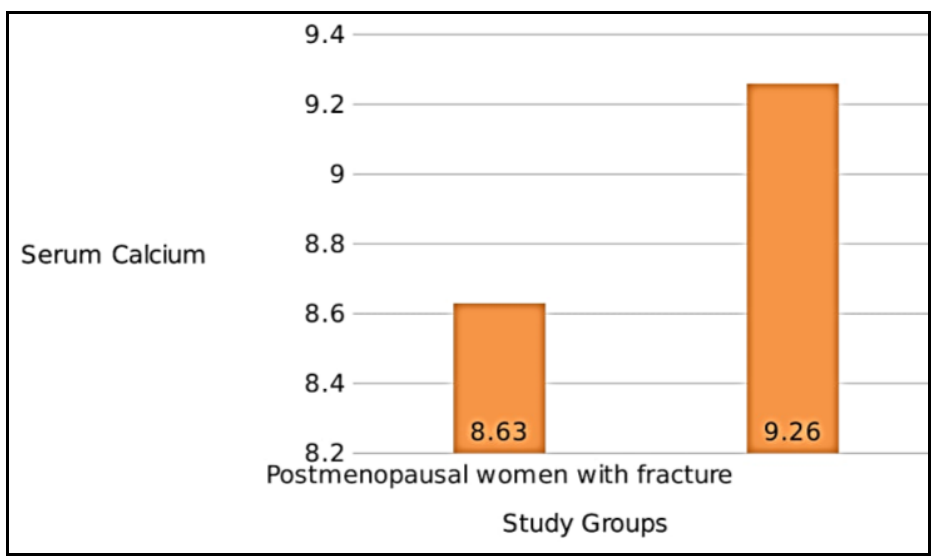

Fig. 2: Mean serum calcium level in the premenopausal and postmenopausal women with fracture 


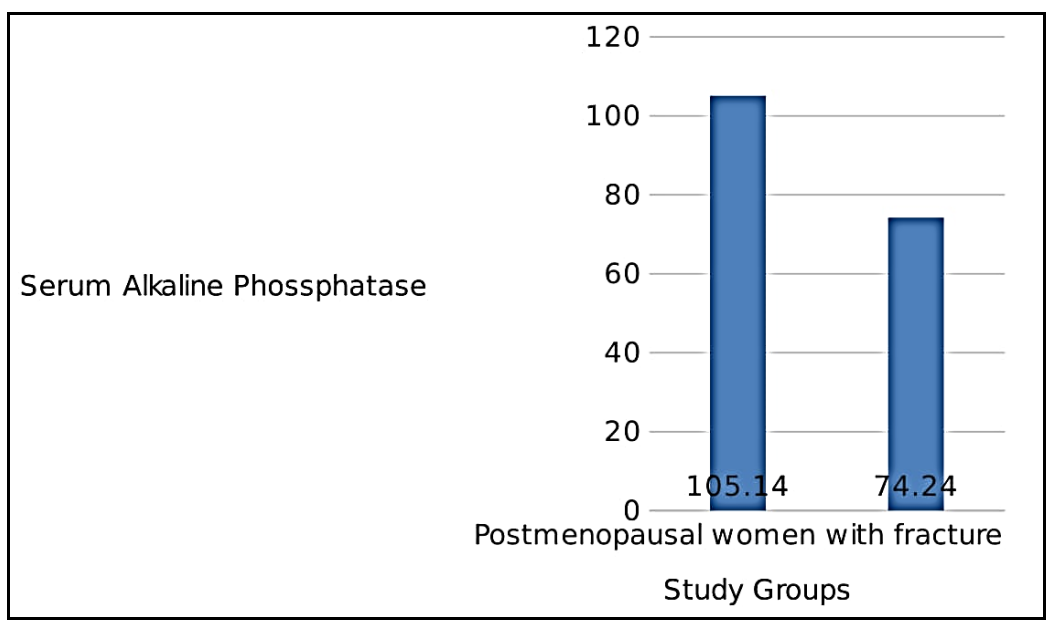

Fig. 3: The mean serum alkaline phosphatase level in the pre and postmenopausal women with fracture groups

\section{Discussion}

In the present study we have demonstrated very low levels of serum osteocalcin in postmenopausal women with fractures as compared to the premenopausal women which is parallel to a study done in Sweden where there was a $20 \%$ decrease in the osteocalcin level among the postmenopausal women with fractures than apparently healthy postmenopausal women. Similarly Pietschmann P et al also showed lower osteocalcin levels in postmenopausal osteoporotic women as compared to postmenopausal women. ${ }^{11}$ However V.S Kalai Selvi et al ${ }^{12}$ and M Beg et $\mathrm{al}^{13}$ differ in their results showing higher serum osteocalcin levels in the osteoporotic women as compared to the premenopausal women and post menopausal women without fractures. In late menopause bone formation is reduced and resorption activity is increased which is reflected by decrease in osteocalcin levels hence explains the fractures in these women even with a trivial fall. In early menopause there is an accelerated osteoclastic activity due to the sudden depletion of estrogen which increases bone resorption, as resorption and formation are coupled and this is reflected in the serum as increased osteocalcin levels. Thus it is a highly sensitive bone formation marker.

There was a highly significant lower mean serum calcium level in postmenopausal women with fractures as compared to the control group, similar to a study done by Surya Prabha Y et al in Andhra Pradesh where there was higher calcium level seen in controls as compared to postmenopausal women with osteoporosis. ${ }^{14}$ Calcium balance plays a critical role to maintain the bone mass. It is a known fact that with increase in age and estrogen deficiency causes excess osteoclastic action with an increase in calcium loss by increasing its excretion and decreasing its absorption.

Protein is an important constituent for the formation of bone and albumin is a sensitive marker of poor nutritional status. In this study the mean serum total protein level was not statistically significant in both the study groups whereas it also showed higher serum albumin levels in the premenopausal women as compared to post menopausal group with fractures and a positive significant correlation of osteocalcin and serum albumin. Similarly an inverse relationship of serum albumin was seen with osteoporosis. Longer duration towards the decrease in its level showed risk of osteoporosis in a study done by Farshad et al. ${ }^{15}$ Rico. H, et al used the levels of transferrin, retinol binding protein and pre albumin in women with osteoporotic hip fractures and found that the concentrations of all the three protein markers were lower in the group of patients who had fractures. ${ }^{16}$ Similarly D' Erasmo et al also showed negative correlation of albumin levels to increase in years of menopause and fractured postmenopausal women. The mechanism of hypoalbuminemia in osteoporosis remains controversial and could be either due to albumin being present in the osteoid and bone matrix. Hence there is a decrease in the bone mass due to hypoalbuminemia or it may directly be linked with release of nuclear factor-Kb, known to activate osteoclasts and decrease osteoblastic activity. ${ }^{17}$

Immediately after a fracture the total serum alkaline phosphatase level increases due to a systemic response of the body to injury or due to the drugs which may have an effect on the liver. Our study hence showed higher serum alkaline phosphatase in postmenopausal women with fracture group than premenopausal women and a negative correlation of alkaline phosphatase to serum osteocalcin was observed even though both are bone formation markers. These findings are consistent with Bowles $\mathrm{S}$ et al, they estimated serum osteocalcin, total and bone specific alkaline phosphatase and found that total alkaline phosphatase increases immediately after fracture but bone specific alkaline phosphatase and osteocalcin decreases. The increase in total serum alkaline phosphatase in their study was due to the increase in the liver iso enzyme of alkaline phosphatase. ${ }^{18}$ 
The serum osteocalcin had a positive significant correlation with BMI. BMI is associated with low bone mineral density and fractures in older women. In this study postmenopausal women with fractures had low mean BMI. This finding is consistent with a retrospective cohort study done by Morin $\mathrm{S}$ et al, ${ }^{19}$ which concluded that low body weight and BMI can predict osteoporosis and are associated with increased fracture risk in women, hence explains the positive correlation in our study between the two parameters.

Limitations: A small sample size was assessed and other bone formation markers should have also been compared to get a clearer picture. DEXA and BMD should have been compared with the osteocalcin level but due to less funding we could not assess these parameters.

\section{Conclusion}

Osteocalcin is a highly sensitive and inexpensive bone formation marker that can be used as a diagnostic or screening tool in post menopausal women for osteoporosis. The decreased serum osteocalcin levels post menopause could thus predict osteoporosis and with timely medical intervention could prevent the risk of osteoporotic fractures thereby decreasing the mortality rate

\section{References}

1. Sudhir S, Dharmendra K and Atil K. Serum osteocalcin as a diagnostic biomarker for primary osteoporosis in women. Journal of Clinical and Diagnostic Research. 2015;9(8):4-7.

2. Neer RM, Arnaud CD, Zanchetta JR, et al. Effect of parathyroid hormone on fractures and bone mineral density in postmenopausal women with osteoporosis. Endocrinal Metab Clin North Am. 32(1);115-134.

3. Kanis JA, Johnell O, Oden A et al. Risk of hip fracture according to the world health organization criteria for osteopenia and osteoporosis. Bone. 27;(5):585-590.

4. Luukinen H, Kakonen SM, Petterson k et al. Strong prediction of fractures among elderly adults by the ratio of carboxylated to total serum osteocalcin. $J$ Bone Mineral Res. 2000;15(12):2473-2478.

5. Bauer, P.J. Estimation of calcium. Annal Biochem. 1981;110:61.

6. Teitz, N.W. Estimation of serum inorganic phosphorous. Clinical guide to laboratory tests, $3^{\text {rd }}$ edition, W.B. Saunders, 1983.
7. Schledusch, H. et al, Alkaline phosphatase liquiColor. Dtsch med wschr. 1974.

8. Gomall A. Estimation of total protein. J Biol chem. 1949;(751): 177 .

9. Dounasa. B.T. et al. Estimation of serum albumin. Cain chim acto. 1971;87:31.

10. Merle B, Delmas PD. Normal carboxylation of circulating osteocalcin. Bone Miner. 1990;11:237-245.

11. Pietschmann P, Resch H, Krexner E, WoloszczukW, Willvonseder R. Decreased serum osteocalcin levels in patients with postmenopausal osteoporosis. Acta Med Austriaca. 1991;18(5):114-6.

12. Kalaiselvi VS, Prabhu K, Ramesh M, Vathsala V. The association of serum osteocalcin with bone mineral density in post menopausal women. J Clin Diagn Res. 2013;7(5):814-816.

13. Beg M, Akhtar N, Alam MF, Rizvi I, Ahmad J. Vitamin D status and serum osteocalcin levels in postmenopausal osteoporosis. Journal, Indian Academy of Clinical Medicine. 2014;15(3-4):172-176.

14. Surya Prabha Y, Ashalata K, Vijaya Babu P.V.S.S, Kusuma Kumari P and Nagamani M. A study of bone markers (Serum Calcium, Serum Phosphorous and Serum Alkaline phosphatase) in postmenopausal women in East Godavari District, Andhra Pradesh, India. IOSR Journal of Dental and Medical sciences. 2015;14(6):1-3.

15. Farsad A, Ka Kit Wong, Baskaran S, Robert J, Ackermann and Subramaniam P. Hypoalbuminemia and Osteoporosis: Reappraisal of a Controversy. J Clin Endocrinol Metab. 2016;101(1):167-175.

16. Rico H, Relea P, Crespo R, Revilla M, Villa LF, Arribas I et al. Biochemical markers of nutrition in type I and type II osteoporosis. J Bone Joint Surg Br. 1995;77(1):148-51.

17. D'Erasmo E, Pisani D, Alessandro ragno, raejntroph N, Claudia L and Acca M. Relationship between serum albumin and bone mineral density in postmenopausal women and in patients with hypoalbuminemia. Hormone and metabolic research.1999;31(6):385-8.

18. Bowles SA, Kurdy N, Davis AM, France MW, Marsh DR. Serum osteocalcin, total and bone-specific alkaline phosphatase following isolated tibial shaft fracture. Ann Clin Biochem. 1996:33:196-200.

19. Morin S, Tsang JF L, Leslie WD. Weight and body mass index predict bone mineral density and fractures in women aged 40 to 59 years. Osteoporosis Int. 2009;20(3):363-370.

How to cite this article: Rai M, Rai T, Rai S, D'Sa J, Parinita K. Serum osteocalcin: A potential biomarker for primary osteoporosis. Int J Clin Biochem Res. 2018;5(3):392-396. 